\title{
Zero-gap materials for future spintronics, electronics and optics
}

\author{
Xiao-Lin Wang ${ }^{1 *}$, Shi Xue Dou' and Chao Zhang ${ }^{1,2}$ \\ University of Wollongong, Australia
}

\begin{abstract}
Electrons carry both charge and spin. The processing of information in conventional electronic devices is based only on the charge of the electrons. Spin electronics, or spintronics, uses the spin of electrons, as well as their charge, to process information. Metals, semiconductors and insulators are the basic materials that constitute the components of electronic devices, and these have been transforming all aspects of society for over a century. In contrast, magnetic metals, half-metals, magnetic semiconductors, dilute magnetic semiconductors and magnetic insulators are the materials that will form the basis for spintronic devices. Materials having a zero-energy band gap are a special class of these materials that exhibit some fascinating and superior electronic properties compared to materials with a non-zero energy gap. This article reviews a range of materials with zero-gap band structures, focusing on materials with quadratic and linear symmetrical dispersions, disorder-induced linear dispersions, asymmetrical linear dispersions and topological insulating states. These materials all have intriguing physical properties and numerous potential practical applications in spintronics, electronics, optics and sensors.
\end{abstract}

$\mathrm{n}$ spite of the extreme complexity and uncountable number of materials and substances that are created in nature, all can be classified quite simply in terms of their conductive behavior into one of three types - insulators, semiconductors and metals - depending on the electronic band structure. It is the electrons carried by the constituent elements and their interactions that determine the structure of a material and its physical properties, such as electrical conductivity, magnetism and superconductivity. The degrees of freedom of electrons in a material determine the material's electronic properties. Making use of the spin of electrons adds one more degree of freedom, which can be utilized to design enhanced electronic devices [1]. The materials that enable us to take advantage of the spin of electrons are primarily those that exhibit magnetic properties. Ferromagnetic and antiferromagnetic metals, as well as semiconductors and insulators, are the materials that form the foundations for metallic, semiconducting and insulating spintronics, respectively, and these materials have been under active investigation by many groups around the world $[2,3]$. The performance of spintronic devices is determined by the degree of spin polarization of free electrons within a material. Electrons are partially polarized in classical ferromagnetic metals, such as iron, cobalt and nickel, yet can be fully polarized in half-metals and certain dilute semiconductors.

Ferroelectric magnets and multiferroic materials, which exhibit coupling between ferroic order parameters, are candidates for use in insulating spintronics. In these materials, the spin (magnetic) dipole and the electric dipole can be mutually manipulated by electric and magnetic fields [4,5]. Figure 1 summarizes the band structures of these various classes of materials. Metals contain a conduction band that is partially filled, which means that the Fermi level $\left(E_{\mathrm{f}}\right)$ penetrates into the conduction band. In contrast, insulators have a band structure in which the conduction and valence bands are separated by a large energy gap $\left(E_{\mathrm{g}}\right)$. Semiconductors exhibit a narrow energy gap, while semi-metals have a small energy overlap between
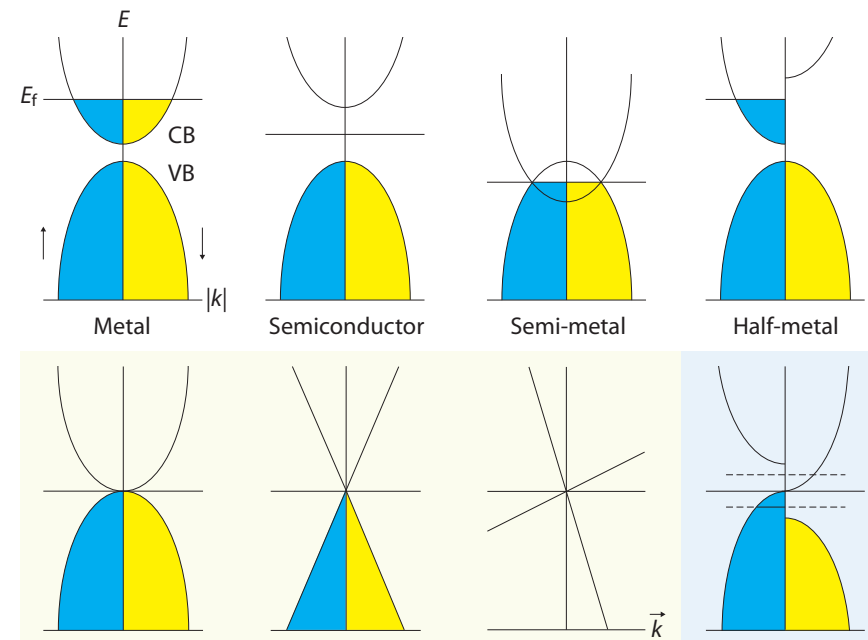

Quadratic
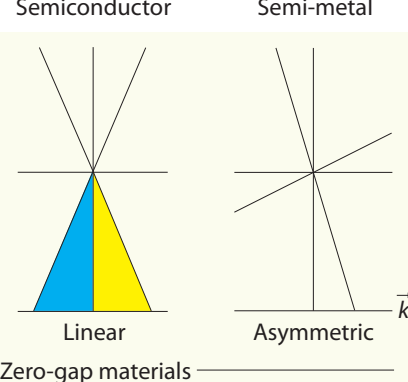

Half-metal

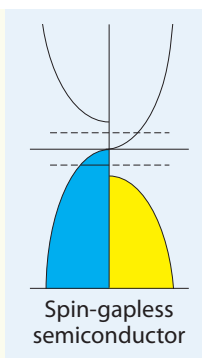

Figure 1. The electronic band structures of various classes of materials. Figures show the energy-momentum ( $E-k)$ dispersions with respect to the Fermi level $\left(E_{f}\right)$. The blue and yellow areas indicate the spin-up and spin-down charge carrier states, respectively. In intrinsic zero-gap materials, the conduction band (CB) and valence band (VB) edges touch at the Fermi level. Graphene is a zero-gap material with linear energy dispersion. The band structure of the spin-gapless semiconductors is spin-dependent.

the conduction and valence bands. Half-metals are metallic in one spin direction and semiconducting in the other [6,7]. Half-metallic antiferromagnets, a newly theorized system predicted in some alloys, perovskite oxides and dilute magnetic semiconductors, have fully 


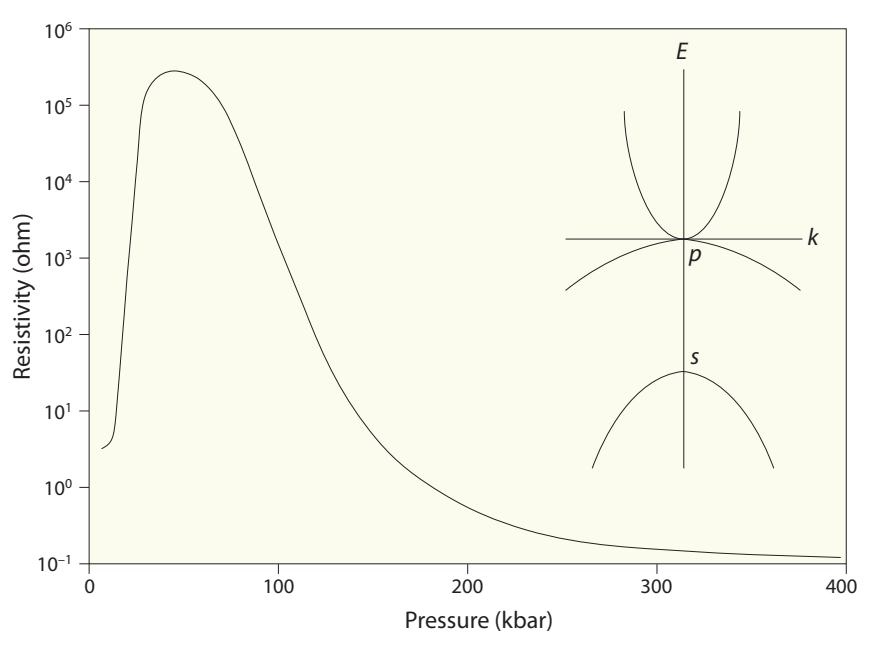

Figure 2. Pressure dependence of resistivity of HgSe. (Inset) Inverted band model (reversed $s$ and $p$ bands) for HgTe and $\mathrm{a}-\mathrm{Sn}$.

spin-polarized conduction electrons but no net magnetization [8-11]. Dilute magnetic semiconductors and half-metals with full electron spin polarization are the most important candidates with respect to spintronics applications $[2,3]$.

If the conduction and valence band edges meet at the Fermi level, the material belongs to a comparatively new class of solids known as gapless semiconductors or zero-gap materials [12]. In these materials, no threshold energy is required to move electrons from occupied states in the valence band to empty states in the conduction band. As a consequence, gapless semiconductors have unique properties including an extreme sensitivity of the band structure to external influences such as pressure or magnetic field. The electron mobility of a gapless semiconductor is $2-4$ orders of magnitude higher than that for classical semiconductors. The very recent and exciting discovery of two-dimensional (2D) gapless graphene has aroused great interest worldwide [13]. The features of zero band gap dispersion distinguish zero-gap materials from all other materials in many respects. As shown in Figure 1, materials with zerogap band structures can have quadratic, linear and asymmetrical energy-momentum $(E-k)$ dispersions, and various states. This article reviews the fascinating and unusual spintronic, electric and optical properties of these materials and of topological insulators, a new class of quantum matter. It is expected that zero-gap materials with exotic band features and electronic states will constitute a new class of materials that will form the basis for next-generation spintronic, electronic and optical devices.

\section{Zero-gap materials with quadratic energy dispersion}

Only a few materials have a zero or very narrow band gap with a quadratic energy-momentum dispersion. Mercury telluride ( $\mathrm{HgTe}$ ) is one of a very few parent compounds in this class that have been studied rigorously since the 1950s [12]. The intrinsic charge-carrier concentration $(n)$ of a zero-gap material varies with temperature $(T)$ according to a power law, $n \propto T^{3 / 2}$. In a conventional semiconductor with a discrete energy gap, $n$ increases exponentially with temperature [12]. The size of the energy gap can be tuned in solid solutions of $\mathrm{Hg}_{1-x} \mathrm{Cd}_{x} \mathrm{Te}$ [14], and for this reason these compounds have been used in infrared light detection and emission applications at wavelengths of $1-30 \mu \mathrm{m}$ in both the civil and military sectors [15]. The energy gap of the system at $4.2 \mathrm{~K}$ changes from $-0.30 \mathrm{eV}$ for undoped $\mathrm{HgTe}$ to zero for compositions of $x=0.15-0.16$ and $1.65 \mathrm{eV}$ for CdTe.

Another material with a quadratic energy-momentum dispersion and near-zero energy gap is a-phase tin (gray tin), which has the same crystal structure as the group-IV elements, such as carbon (diamond), silicon and germanium. The energy gap is less than $0.08 \mathrm{eV}$ [16], and the conduction-band minimum and valence-band maximum coincide at $k=0$ [17]. An inverted band model in which the p-symmetry band is at higher energy than the $s$-symmetry band, the reverse of the typical order has been proposed to explain the characteristics of transport, photoconductivity and light reflection for zero-gap $\mathrm{HgTe}$ and $\alpha-S n$ (Figure 2) [18]. The zero-gap state can be attained at certain values of external isotropic effects, such as hydrostatic pressure, temperature and degree of alloying, that do not change the crystal symmetry. As a result of such accidental degeneracy, certain compositions of solid solutions of $\mathrm{Pb}_{1-x} \mathrm{Sn}_{x} \mathrm{Te}, \mathrm{Pb}_{1-x} \mathrm{Sn}_{x} \mathrm{Se}$ and $\mathrm{Bi}_{x} \mathrm{Sb}_{1-x}$ are zero-gap materials [12].

As both the conduction and valence band edges meet at the Fermi level, the transport and optical properties of zero-gap materials are extremely sensitive to physical perturbations. For example, the zero-gap band can be modified by electric and magnetic fields, as well as by pressure and chemical inhomogeneity. Electric fields influence the charge carriers, and the magnetic field changes the size of the gap, which in turn changes the density of charge carriers. An increase in the mean electron energy under an external electric field leads to a non-linear current-voltage curve. An energy gap can be opened by the application of a magnetic field and external pressure, and the zero-gap state can be reinstated at higher pressure, resulting in more than 4-6 orders of magnitude of variation in resistivity [12].

In zero-gap mercury selenide ( $\mathrm{HgSe})$, another well-studied gapless material, the longitudinal magnetoresistance increases sharply when the crystal is subjected to a magnetic field aligned parallel to the direction of current flow. This component of magnetoresistance increases exponentially with magnetic field strength. The resistivity also varies remarkably with pressure, as shown in Figure 2. Pressure induces a transition in $\mathrm{HgSe}$ from a zero-gap material to a semiconductor with a discrete energy gap, and eventually to a metal [12]. Doping with magnetic ions, such as manganese or iron, can lead to giant magnetoresistance in $\mathrm{HgTe}$ or $\mathrm{HgSe}$ [19], making doped $\mathrm{HgSe}$ a suitable material for magnetoelectronics and magnetic-field sensor applications. The energy gap in manganese-doped $\mathrm{HgTe}\left(\mathrm{Hg}_{1-x} \mathrm{Mn}_{x} \mathrm{Te}\right)$ varies linearly with manganese content up to manganese concentrations of as much as $x=0.2$. In zero-gap $\mathrm{HgMnTe}$, the transverse magnetoresistance first increases sharply with increasing magnetic field, and then falls rather abruptly. The longitudinal magnetoresistance, however, decreases monotonically. This evolution in magnetoresistance with magnetic field strength is caused by magnetic field-induced band adjustments [12]. HgMnTe and HgMnSe change from a paramagnetic state to a spin-glass state at a critical temperature $\left(T_{\mathrm{s}}\right)$ as a result of the collapse of the antiferromagnetic interaction in the face-centered cubic lattice. Thus, in addition to their use in infrared photodetectors, zero-gap $\mathrm{HgTe}$ and $\mathrm{HgSe}$ are also useful as Hall and magnetoresistance sensors and as spin photoconductors [12]. The physics and applications of these classical zero-gap materials have been discussed in great detail in Ref. 12.

Generally speaking, the zero-gap state can be obtained in any narrow-band semiconductor through either chemical doping or application of external pressure. It should be noted that all narrowband or zero-gap materials are non-oxide-based III-V or II-V compounds that contain toxic elements such as mercury and tellurium. It is therefore desirable to find non-toxic zero-gap materials.

\section{Zero-gap materials with linear energy dispersion}

\section{Graphene}

Recent progress in isolating single sheets of graphite [20-24] has sparked interest in graphene-based nanoelectronics. Experiments have already demonstrated the anticipated physics of monolayer graphene, including electron-hole symmetry, a half-integer quantum Hall effect $[21,22]$, finite conductivity at zero charge-carrier concentration [21], and strong suppression of weak localization [23-27]. By further confining the electrons in the graphene plane, one-dimensional 


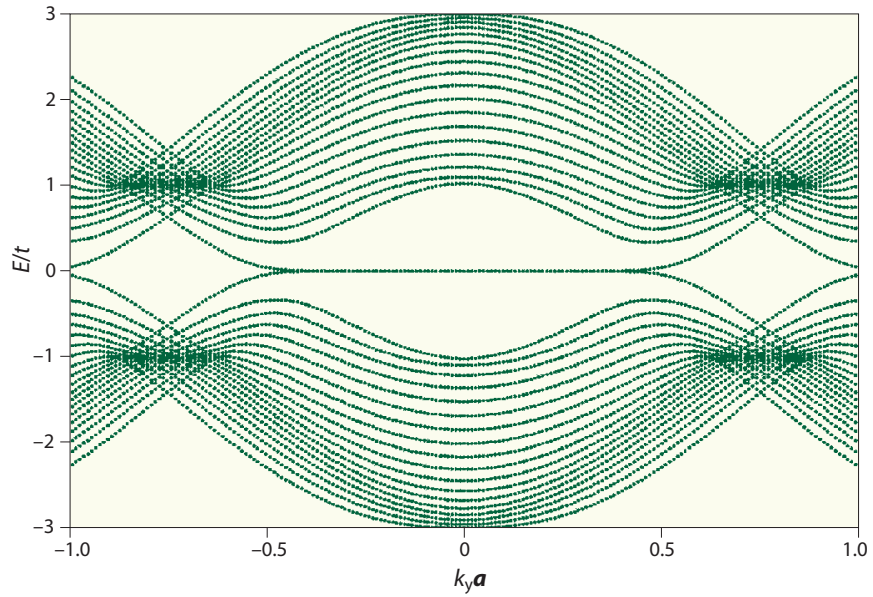

Figure 3. The gapless structure of a 20-unit zigzag graphene nanoribbon is robust under a constant magnetic field. The field strength is equivalent to 1/00 flux quanta per unit cell. $k_{y}$ is the wavevector along the long ribbon direction, $\boldsymbol{a}$ is the unit vector of the honeycomb lattice, $E$ is the electronic energy and $t$ is the nearest-neighbor hopping bandwidth in graphene.

graphene structures (graphene nanoribbons) can be obtained. This is a unique class of nanosystem in which one-dimensional gapless energy dispersions can be achieved. It has been suggested that graphene nanoribbons could be used as field-effect transistors [28]. The remarkable properties of graphene-based materials make them promising building blocks for technological applications in molecular electronic and optoelectronic devices.

In graphene, the conduction and valence bands touch at isolated points ( $\mathrm{K}$ and $\mathrm{K}^{\prime}$ ) in the first Brillouin zone (the reciprocal crystal lattice). Undoped graphene is a gapless semiconductor with a vanishing density of states at the Fermi level, and the energy-momentum dispersion is linear (Figure 1). As such, the electrons in graphene travel at a constant speed of 0.0025 times the speed of light, much faster than in typical semiconductors. The low-energy electronic states in graphene at the $\mathrm{K}$ and $\mathrm{K}^{\prime}$ corners of the Brillioun zone can thus be described by the relativistic massless Dirac equation, and this relativistic description has been confirmed in quantum Hall studies [29]. These and subsequent studies have provided theoretical insight into the exotic transport [30-32], magnetic correlation [33-35] and dielectric [36] properties of graphene.

In graphene nanoribbons, the form of the gapless dispersion (linear or quadratic) and the location of the gapless point ( $\Gamma$ point or $K$ point $)$ are determined by the width and chirality of the ribbon and are thus predictable. Graphene ribbons have various morphologies, which can be defined by the indices $p$ and $q$ [37]. Zigzag ribbons $(p, 0)$ are gapless materials with a quadratic energy dispersion at the zero-gap point, which occurs at the $\mathrm{K}$ point. The gapless band structure of zigzag ribbons is robust under an applied magnetic field (Figure 3). Armchair ribbons can be gapless or have a finite energy gap depending on the ribbon width. There also exists a class of armchair nanoribbons $(3 p-1,1)$ with gapless energy dispersion at the $\Gamma$ point. This class of ribbons can have an unusually strong response to a finite magnetic field in the lowfrequency regime [38].

The gapless energy spectrum of electrons and holes in graphene can lead to very specific optical properties. Certain types of bilayer ribbons with zero gap can have very high optical response [39]. Very recently, it has been demonstrated experimentally that both the optical and direct-current conductance have constant values [40,41], as shown in Figure 4. As a result, the transmission coefficient [42] of graphene in the frequency range of $700-400 \mathrm{~nm}$ is very close to a constant value. It is anticipated that the optical spectrum induced by elementary electronic excitations can be used to determine the electronic properties

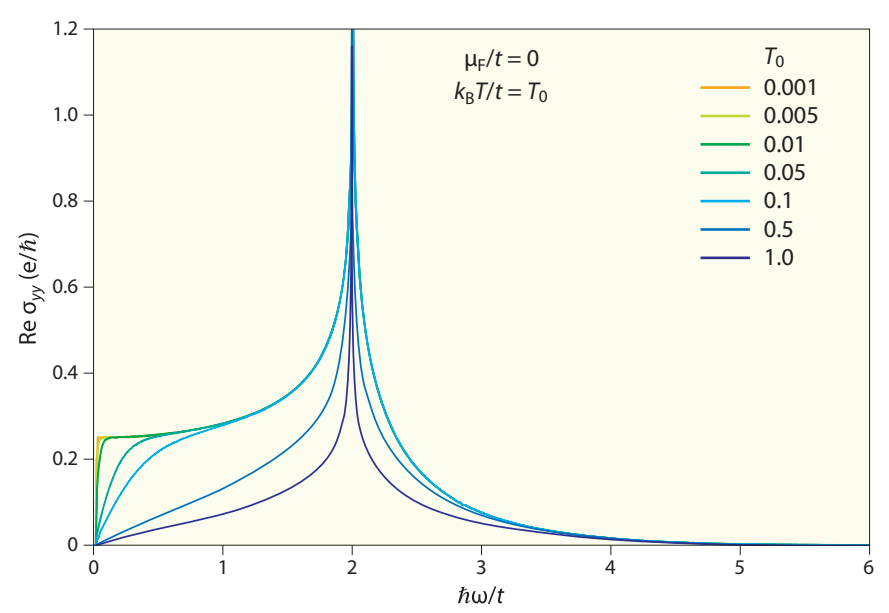

Figure 4. Frequency dependence of conductance $\left(\sigma_{y y}\right)$ of gapless graphene. The $x$ axis denotes the photon frequency $(\hbar \omega)$ normalized by the hopping bandwidth $(t)$. At low temperature, the conductivity is on the order of $\pi e^{2} / 2 h$, which can be viewed as a frequency-dependent analog of minimum conductivity [32] as a direct consequence of the zero-gap energy dispersion at the $\mathrm{K}$ point.

of graphene. It is well known that optical conductivity is one of the central quantities that determine almost all the optical properties of an electron and/or hole system. For the case in which an optical transition is induced mainly by a dielectric response of the carriers through carrier-carrier interaction, the optical conductivity can be obtained simply from the Kubo formula, in which the current-current correlation is primarily attributable to carrier interactions with a weak external light field.

The optical properties of a zero-gap system at low frequencies can be tuned or enhanced by spintronic effects, such as carrier spin-orbit coupling. Around the $\mathrm{K}$ point, the energy dispersion is isotropic, and momentum $k$ corresponds to the energy of the laser field. This leads to a node in the optical transition, arising from the fact that if the light is polarized perpendicular to the electron wave vector, no absorption is possible because of the linear energy dispersion. The spin-orbit interaction can remove this restriction because the energy is no longer strictly proportional to $k$. The total absorption in the important terahertz frequency range can be enhanced by up to $100 \%$, opening the way for spintronic applications of zero-gap materials.

There is an intrinsic relation between the zero-gap energy dispersion and nanomagnetism. For example, all armchair graphene nanoribbons are nonmagnetic, and all zigzag graphene nanoribbons have antiferromagnetic order $[33,43]$. It has been shown that the ground-state magnetic ordering within a single nanoribbon, nanodot or nanohole is consistent with the theorem of itinerant magnetism in a bipartite lattice within the one-orbital Hubbard model [44].

Under a constant magnetic field, the gapless energy dispersion in graphene nanoribbons splits into magnetic subbands. If only nearestneighbor coupling is considered, the lowest magnetic subband is at zero energy. In other words, the lowest energy state, under magnetic quantization, for a linear gapless system, is zero. A direct consequence of this is the Hall plateau at zero field. If the next-nearest neighbor is included, the zero-gap point will be shifted to negative energy. In this case, the magnetic subband structure is asymmetric [45].

For graphene nanoribbons, the magnetic susceptibility is strongly dependent on the width and orientation of the ribbon. The edge states of the ribbons have a dominant effect on the low-field susceptibility. For both zigzag and armchair ribbons, the susceptibility increases rapidly as the ribbon width increases. This is mainly due to the fact that as the ribbon width narrows, the edge states become increasingly important. However, these edge states do not carry an orbital magnetic moment at low fields. The gapless dispersion in this case will play 


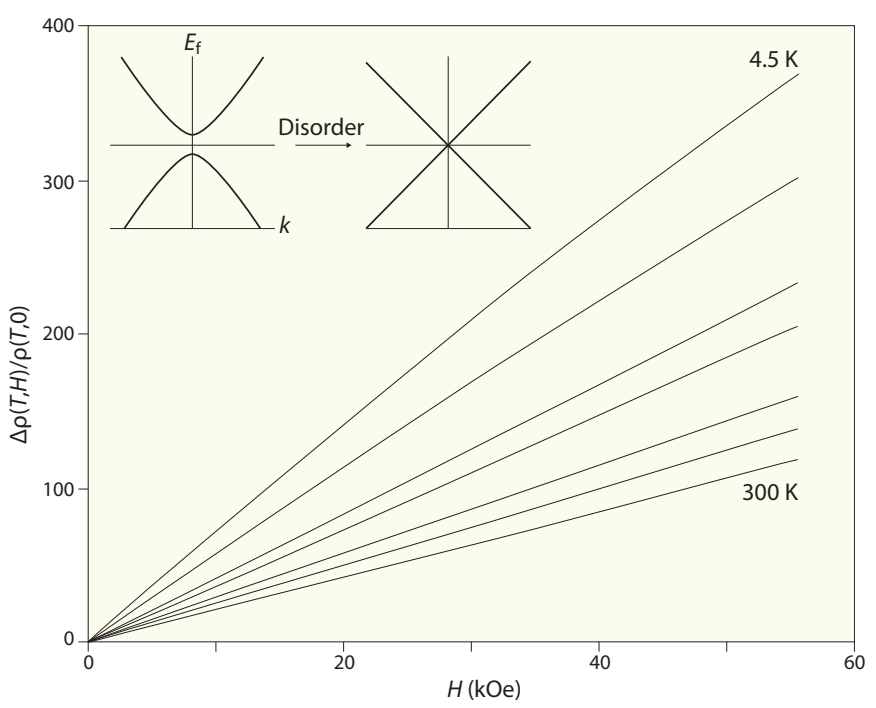

Figure 5. Quantum linear magnetoresistance in highly disordered $\mathrm{Ag}_{2 \pm \delta} \mathrm{Se}$. (Inset) Band structure of a narrow-gap semiconductor (left), which can be changed to a zero-gap structure with linear energy dispersion (right) due to disorder. Adapted from Ref. 46.

a crucial role in the orbital magnetization. Systems with gapless dispersions at the $\mathrm{K}$ and $\mathrm{K}^{\prime}$ points have much stronger magnetization. For ribbons of the same width, the magnetic susceptibility depends on the chirality of the ribbon. For ribbon widths of $50 b(b=1.42 \AA$, the carbon-carbon distance), the zero-field susceptibility of the zigzag ribbon is around $20 \%$ stronger than that of the armchair ribbon. If the ribbon width is $20 b$, this difference is more than $100 \%$.

\section{Disorder-induced zero-gap materials with linear dispersion}

In 1997, surprisingly high giant positive and linear magnetoresistance was observed in two non-magnetic narrow-band semiconductors, $\mathrm{Ag}_{2 \pm \delta} \mathrm{Se}$ and $\mathrm{Ag}_{2 \pm \delta} \mathrm{Te}$, which have highly disordered stoichiometry [46]. Such linear magnetoresistance can persist from extremely low fields up to very high fields without any sign of saturation and is weakly temperature-dependent at elevated temperature [46]. In order to explain this astonishing phenomenon in non-magnetic materials, Abrikosov proposed that the disorder induced by non-stoichiometry can transform a narrow-gap semiconductor into a zero-gap state with a Dirac-cone-like linear energy spectrum in both the valence and conduction bands [47] (see Figure 5, inset). Although differing in dimensionality, this is the same structure as the zero-gap band structure with linear energy dispersion for graphene (Figure 1). Only one Landau level with a linear energy spectrum is assumed to participate in the conductivity for such a zero-gap state induced by stoichiometry disorder.

Abrikosov developed a generic quantum description for galvanomagnetic phenomena, as given by [47]

$$
\rho_{x x}=\rho_{y y}=\frac{N_{i} H}{\pi n^{2} e c} \propto H
$$

where $\rho_{x x}$ and $\rho_{y y}$ are the transverse components of magnetoresistance, $n$ is the density of electrons, and $N_{i}$ is the concentration of static scattering centers. This quantum magnetoresistance is linear down to very small fields, and is positive, non-saturating, and more importantly, independent of temperature. According to this model, band-gap tuning for silver chalcogenides [48] induces a change of sign in the Hall coefficient and a linear field dependence of the magnetoresistance. It should be noted that the magnitude of the fluctuations in mobility caused by disorder rather than the mobility itself can also account for the linear magnetoresistance [49]. The zero-gap structure with linear magnetoresistance pertains to materials with three-dimensional crystal structures rather than one-atom-thick graphene or other two-dimensional materials. Therefore, the transport and magnetotransport properties of three-dimensional materials with a zero-gap state differ greatly from those of graphene. Such zero-gap materials with linear dispersion can be obtained through the introduction of stoichiometric disorder, or through external perturbations in semimetals or semiconductors with a narrow gap. Very recently, colossal linear magnetoresistance was successfully observed in antimony-rich InSb. It was found that the magnetoresistance increases linearly under both extremely low and high fields, and increases by up to $500 \%$ under a magnetic field of $15 \mathrm{~T}$ without saturation [50]. It is believed that the disorder induced by non-stoichiometry in $\mathrm{InSb}_{1+x}$ leads to a zero-gap state with linear dispersion. The linear magnetoresistance under both low and high fields makes such disorder-induced zero-gap materials with linear energy dispersion very useful for low and high magnetic field sensor applications. It is expected that the quantum magnetoresistance could be present in other three-dimensional narrow-gap semiconductors with high stoichiometric disorder.

\section{Zero-gap materials with asymmetrical energy dispersion}

The band structure of these materials results in an asymmetrical energy-momentum dispersion (Figure 1). This situation is very unusual, but could exist in real materials. A zero-gap structure with a linear asymmetrical energy dispersion was found to exist theoretically in a quasi-two-dimensional organic charge-transfer conductor, $\alpha$-(BEDT-TTF $)_{2} \mathrm{I}_{3}$ salt [51]. This organic conductor is one of the members of the (BEDT-TTF) ${ }_{2} \mathrm{I}_{3}$ family, which consists of conductive layers of bis(ethylenedithio)tetrathiafulvalene (BEDT-TTF) molecules and insulating layers of $\mathrm{I}_{3}{ }^{-}$ions. This two-dimensional organic conductor shows a metal-insulator transition at $135 \mathrm{~K}$ at ambient pressure [52] and changes to a metallic, superconductive or zero-gap state with a Dirac-cone-like linear dispersion under different uniaxial pressures, even at low temperature [53]. Pressure along the $a$ axis can induce significant change in the resistance of this organic conductor, as shown in Figure6. First-principles calculations have indicated that this compound has a unique band structure near the Fermi level, called the two-dimensional anisotropic Dirac cone dispersion [43], $E= \pm v_{\mathrm{f}} K_{\mathrm{c}}$, where $v_{\mathrm{f}}$ is the Fermi velocity and $K_{\mathrm{c}}$ is the wavenumber on the $a-b$ plane. Dirac cone dispersion occurs at a general $k$ point, as well as at the Fermi level, instead of at a high-symmetry point as in the case of graphene (see Figure 6, inset) [51].

In comparison with graphene, $\alpha$-(BEDT-TTF $)_{2} \mathrm{I}_{3}$, which has a zero-gap state with asymmetrical linear energy dispersion, exhibits the following electrical and magnetic properties: a linear density of states, which results in a $T^{2}$ dependence of carrier density up to $100 \mathrm{~K}$; low carrier density $\left(10^{15} / \mathrm{cm}^{2}\right)$ and high mobility $\left(3 \times 10^{5} \mathrm{~cm}^{2} /(\mathrm{V} \cdot \mathrm{s})\right)$ resulting in a resistivity that is independent of temperature; and positive giant magnetoresistance. The transport properties of zero-gap $\alpha$-(BEDT-TTF $)_{2} \mathrm{I}_{3}$ are not the same as has been seen in graphene. As shown in Figure 6, there are two resistance states; a low-resistance state related to the Dirac cone carrier system [54], and a high-resistance state likely to be caused by effects on charge carriers from band tuning by the magnetic field. However, due to the unique asymmetrical linear energy dispersion, some unique optical properties can be expected. Under thermal or light excitation, the excited electrons and holes both have positive $k$ but different energies and velocities. This means that the light excitation can cause electrons and holes to have different masses and undergo transport at different speeds, leading to current flow. For the asymmetrical zero-gap band structure, the energy dispersion could also be quadratic, the same as for the quadratic symmetrical zero-gap structure. In this case, the carrier's mass and mobility would be much greater, but lower than in asymmetrical zero-gap materials. 


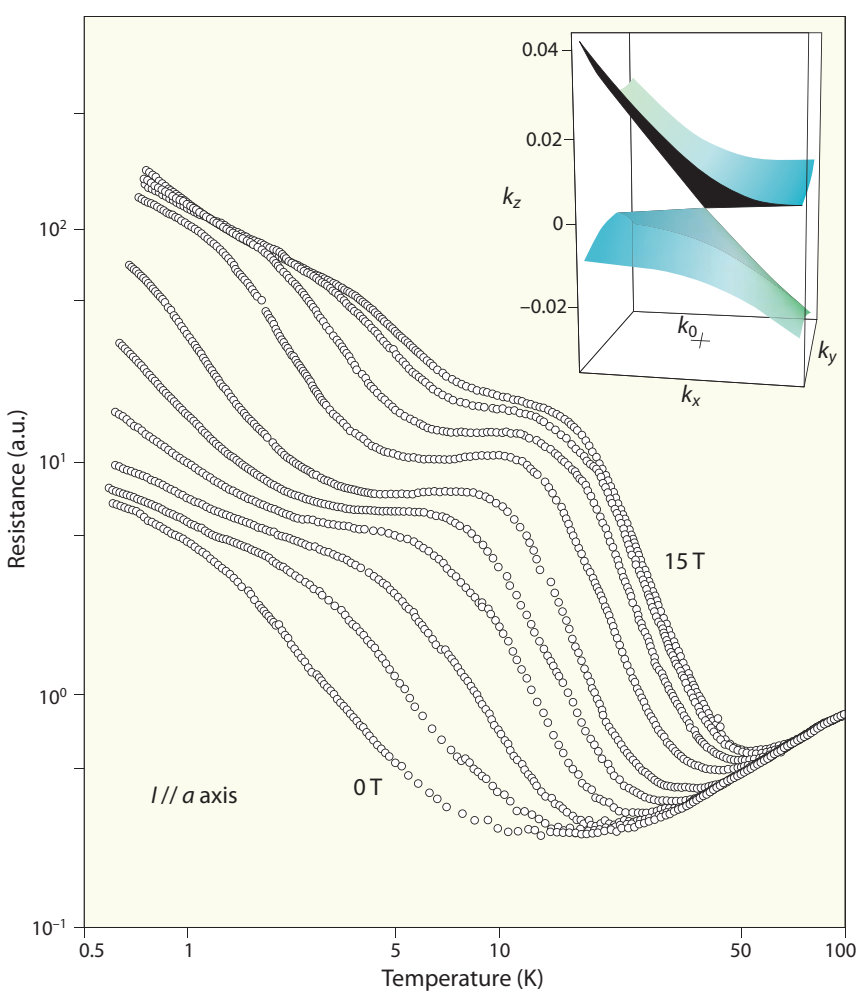

Figure 6. Temperature dependence of a-(BEDT-TTF) $)\left._{2}\right|_{3}$ resistance under various magnetic fields. Modified after Ref. 53 (๔ 2006 JPSJ). (Inset) Three-dimensional asymmetrical linear zero-gap band structure. Modified after A. Kobayashi, S. Katayama, K. Noguchi, Y. Suzumura, J. Phys. Soc. Jpn 73, 3135 (2004) (๔ 2004 JPSJ).

So far, a-(BEDT-TTF $)_{2} \mathrm{I}_{3}$ is the only compound showing both a zero gap and an asymmetrical linear energy dispersion. It might be fruitful to search for more candidates among the many other twodimensional inorganic and organic semiconductors and conductors. Fascinating magnetic and exciting optical properties, in particular, are expected.

\section{Spin-gapless materials}

Inspired by the unique superior properties of the zero-gap materials, the concept of a spin-gapless semiconductor has been proposed [55]. Such a material could have four possible band structure configurations with spin gapless features (see Figure 7) based on the zero gap state and either quadratic or linear energy-momentum dispersions [55]. In the first case (Figure 7(a)), there is a gap between the conduction and valence bands in the respective spin-up and spin-down channels, but zero gap between the spin-up electrons in the valence band and the spin-down electrons in the conduction band. In the second case (Figure $7(\mathrm{~b})$ ), the spin-up channel is gapless, while the top of the spin-down valence band touches the Fermi level and is separated from the corresponding conduction band by a gap. In the third case (Figure 7(c), the spin-up channel is gapless, while the spin-down channel is semiconducting and the top of the spin-down valence band touches the Fermi level. In the fourth case (Figure $7(\mathrm{~d})$ ), one spin channel is gapless, and the other spin channel is semiconducting and neither band of the semiconducting channel touches the Fermi level.

The spin-gapless semiconductors have a number of important features: 1) No energy is required to excite electrons from the valence band to the conduction band, the same as in gapless semiconductors and graphene. 2) The excited charge carriers, both electrons and holes, can be $100 \%$ spin-polarized simultaneously. 3) Using the Hall Effect, fully spin polarized electrons and holes can be easily separated (spinHall effect or field-induced spin-filtering effect). 4) The spin-up and

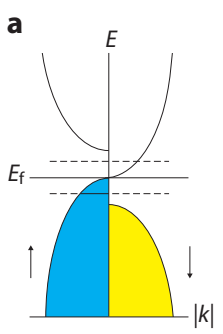

b

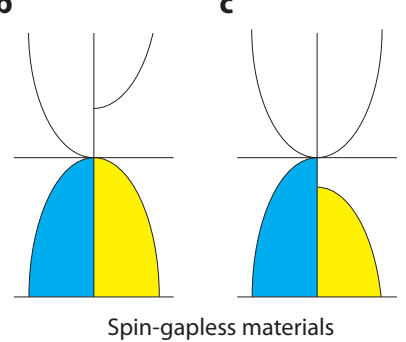

d

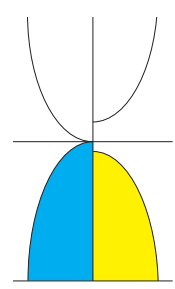

Figure 7. Four possible band structures of spin-gapless semiconductors. See text for details.

spin-down electrons and holes, with both full spin polarization and tunable carrier concentrations, can be easily manipulated in their respective channels by shifting the Fermi level through gate voltage control. As a result of these features, the physical properties of the spingapless semiconductors are very sensitive to external influences such as pressure, electric field, magnetic field, electromagnetic radiation and impurities. Devices based on spin-gapless semiconductors with full spin polarization could be used over a very wide temperature range depending on the size of the gap. The advantages of gapless electronic structures with full spin polarization could lead to novel physics, novel phenomena and new applications, such as new or improved spintronic, electronic, magnetic, optical, mechanical and chemical sensor device applications. In addition to these features, the photons emitted by combination of spin-polarized electrons and holes should be fully polarized, potentially allowing spin-gapless semiconductors to be used as self-polarizers.

One of the possible candidates for such a material is cobalt-doped $\mathrm{PdPbO}_{2}$ [56]. The un-doped compound shows a weakly temperaturedependent resistivity, a feature that possibly indicates a narrow band gap [57]. Band structure calculations based on a unit cell consisting of one cobalt and three palladium atoms indicate that the cobalt-doped $\mathrm{PdPbO}_{2}$ has a zero-band-gap feature similar to that shown in Figure 7(a). The spin-up band touches the spin-down conduction band at the Fermi level. Cobalt-doped $\mathrm{PdPbO}_{2}$ films fabricated by pulsed laser deposition exhibit an anomalous resistance in relation to temperatures, current and magnetic field [56].

The resistivity of the film depends strongly on the magnitude of the electrical current and shows many surprising features: 1) A metal-insulator (or semiconductor) transition is clearly seen, and is more pronounced than has been observed in undoped bulk $\mathrm{PbPdO}_{2}$ samples [57]. 2) The metal-insulator transition temperature $\left(T_{\mathrm{MI}}\right)$ decreases with increasing electrical current, and the variation becomes more prominent at higher currents. 3) The resistivity decreases with increasing current below $T_{\mathrm{MI}}$, but remains constant above $T_{\mathrm{MI}}$. 4) There is a second transition immediately after the resistance becomes saturated (at approximately $1 \mathrm{~mA}$ ). 5) For small currents, the values of the resistivity in the semiconducting/insulating state are almost four orders of magnitude higher than for the metallic state.

Colossal electroresistance, giant magnetoresistance and colossal magnetoresistance are three phenomena present in strongly correlated electron systems that have been puzzling the solid-state physics community ever since they were discovered. The current-dependence of $T_{\mathrm{MI}}$ is similar to what has been seen in manganites, that is, resistance is suppressed at high current $[58,59]$.

As shown in Figure 7(a), a slight shift of the Fermi level could change the conductivity of the material significantly, as has been seen in graphene. This could be the reason for the giant electroresistance that occurs in $\mathrm{PbPd}_{0.75} \mathrm{Co}_{0.25} \mathrm{O}_{2}$ films and could also be an alternative interpretation for the electroresistance observed in colossal magnetoresistance materials, in which electroresistance occurs at the semiconducting-metallic transition where a zero-gap state should exist. 

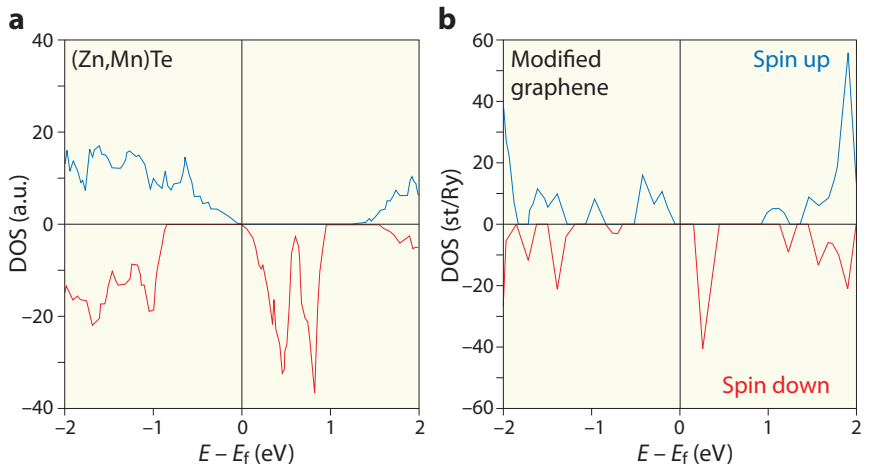

Figure 8. Density of states of (a) manganese-doped ZnTe and (b) graphene with triangular holes. Adapted from (a) L. M. Sandratskii, P. Bruno, J. Phys.: Condens. Matter 15, L585 (2003) (๔ 2003 IOP) and (b) Ref. 60 (reproduced with permission, @ 2008 Springer-Verlag Gmbh).

The spin-gapless semiconductors are expected to open up many prospects for practical applications. Generally, all applications for gapless semiconductors could also be suitable for spin-gapless semiconductors, including spin detectors and generators for electromagnetic radiation over a wide range of wavelengths based on spin photoconductivity. Spin photodiodes and spin image detectors with high mobility of fully spin-polarized carriers might also be feasible.

It should be possible to realize the spin-gapless features in a wide range of other gapless and narrow-band oxide and non-oxide semiconductors, ferromagnetic and antiferromagnetic semiconductors and oxides, and even conductive ferromagnetic oxides and non-oxides with appropriate elemental substitutions. Graphene with various geometries and chemical substitutions is also an obvious target. Manganese-doped $\mathrm{ZnTe}$, in particular, has a density of states that clearly shows a spin-gapless feature. In this material, the top of the valence band of the spin-up electrons and the bottom of the conduction band of spin-down electrons are in contact exactly at the Fermi level (Figure 8). Spin-up and spin-down bands near the Fermi level can also be seen in the density of states for graphene with triangular holes [60]. The gap between the spin-up and spin-down electrons is very narrow and can be tuned to zero with appropriate chemical doping or external influences. The presence of a narrow gap between the spin-up and spindown electrons is actually useful for room-temperature applications. In both systems, spin-up and spin-down electrons and holes with tunable carrier concentrations can be generated easily either by excitation or shift of the Fermi levels.

\section{Zero-gap materials with topological insulator-metal band structures}

It is not naïve to imagine a material that is both insulating and conductive. Such a material can be artificially realized in the form of a thin layer at the interface of two insulators [61]. The interface between two insulating compounds, such as $\mathrm{SrTiO}_{3}$ and $\mathrm{LaAlO}_{3}$ [61], shows surprisingly high electrical conductance, superconductivity and magnetic effects, with a rich electric phase diagram [62-67]. The band structure of such a system should have both three-dimensional insulating and two-dimensional conducting features, as shown in Figure 9(a). The gap originates from the three-dimensional bulk insulator, while the band crossing the Fermi level derives from the metallic state at the interface/surface. Metallic states could also be found at the surface of certain conventional insulators. However, the surface states of a classical three-dimensional insulator are very fragile and can be easily destroyed by geometry and chemistry. Topological insulators are a new class of material or quantum state of matter in a

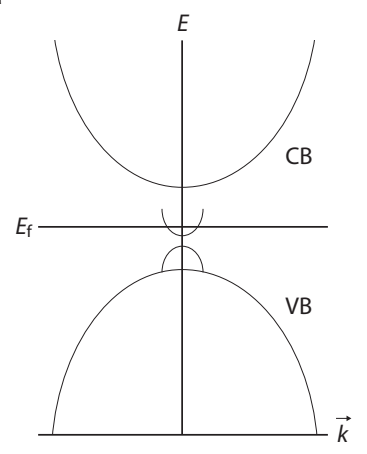

b

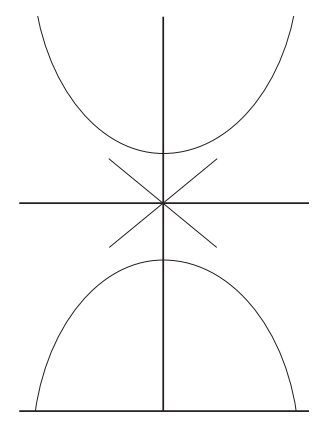

Figure 9. The electronic band structures of topological insulators, a new class of quantum matter with (a) a robust metallic state at the surface/edge and insulating properties in the bulk/surface, and (b) a conductive state at the surface or edge with zero gap and the same linear energy dispersion as graphene.

which a robust metallic state is realized at the surface/edge of an insulator that acts as an insulator in the bulk/surface and a conductor at the surface/edge [68]. The conductive state of the surface or edge has a zero gap (Figure 9(b)) with the same Dirac fermion linear energy dispersion as graphene $[69,70]$. The band crossing the edge or surface is protected by time-reversal [71], and therefore cannot be removed by impurities, yet the Dirac cone can be shifted by changes in the Fermi level. The subject of topological insulators has attracted great attention in condensed matter physics. Two-dimensional topological insulators such as $\mathrm{HgTe} / \mathrm{CdTe}$ quantum wells exhibit a quantum spin Hall effect [69] in the vicinity of the Dirac point. In these systems, charge carriers with opposite spins move without dissipation in opposite directions on a given edge [69]. A three-dimensional topological insulator has a bulk insulating gap with gapless surface states inside the bulk gap [72-75].

Three-dimensional topological insulators with conducting surface states could exist in three-dimensional $\mathrm{HgTe}$ under strain [76], and also in $\mathrm{Bi}_{1-x} \mathrm{Sb}_{x}[77,78]$ and $\alpha-S n$ [77]. Very recently, the stoichiometric systems $\mathrm{Bi}_{2} \mathrm{Te}_{3}, \mathrm{Bi}_{2} \mathrm{Se}_{3}$ and $\mathrm{Sb}_{2} \mathrm{Te}_{3}$, which are well-known thermoelectric materials, have been theoretically predicted to be three-dimensional topological insulators with surface states consisting of a single Dirac cone at the $\mathrm{G}$ point [79-81]. Both $\mathrm{Bi}_{2} \mathrm{Te}_{3}$ and $\mathrm{Bi}_{2} \mathrm{Se}_{3}$ have been experimentally determined to be three-dimensional topological insulators. The Fermi surface contains only one surface state, while the transport properties of $\mathrm{Bi}_{2} \mathrm{Se}_{3}$ are dominated by topological effects. Since the band gap is large, the material can be considered to be a room-temperature topological insulator without alloying disorder. $\mathrm{Bi}_{2} \mathrm{Te}_{3}$ and $\mathrm{Bi}_{2} \mathrm{Se}_{3}$ are distinctive and have advantages over both $\mathrm{Bi}_{0.9} \mathrm{Sb}_{0.1}$ and graphene. The large bulk gaps $(0.16 \mathrm{eV})$ imply great potential for possible high-temperature spintronics applications in a three-dimensional system. Unconventional spin textures were observed in $\mathrm{Bi}_{1-\mathrm{x}} \mathrm{Sb}_{\mathrm{x}}$ three-dimensional topological insulator and are expected to be present in three-dimensional $\mathrm{Bi}_{2} \mathrm{Te}_{3}$, $\mathrm{Bi}_{2} \mathrm{Se}_{3}$ and $\mathrm{Sb}_{2} \mathrm{Te}_{3}$ topological insulators as well. The robust zero-gap states in three-dimensional topological insulators are expected to have novel applications in spintronics, as they provide a route to spin-charge separation in higher dimensions and may allow other novel magnetic and electronic properties to be realized [82].

A quantum spin Hall effect could be expected in the new transition-metal oxide $\mathrm{Na}_{2} \mathrm{IrO}_{3}$ [83], which with a honeycomb lattice structure has been theoretically predicted to be a room-temperature quantum spin Hall insulator. At lower temperatures, the compound is antiferromagnetic at its edges and enters an antiferromagnetic phase in the bulk. Although the topological quantum states of matter are very rare, their unique topological band structure now offers a new opportunity to design a new class of materials and to test some of the profound physics that such systems exhibit. 
Full spin polarization could easily be realized in a spin-gapless material with the band structure shown in Figures $7(a)$ and (d). Under external excitation, the excited spin-down electrons and spin-up holes are fully polarized. Using the Hall effect, one can easily separate fully spin-polarized electrons and holes. The polarized holes and electrons can move to the two surfaces or edges of a two- or threedimensional sample. This spin Hall effect differs from that discovered in topological HgTe quantum wells, in which the spin at the edges of wells can be aligned without a magnetic field [69].

\section{Conclusions}

Materials having a zero or very narrow band gap are a special class of materials that exhibit fascinating and superior electronic, magnetic and optical properties compared to materials with an energy gap. The discovery of zero-gap graphene has generated great interest in the search for new classes of zero-gap materials, and some classical zero-gap materials have been revisited. Zero-gap materials have several different types of energy-momentum dispersions around the energy minimum: symmetrical quadratic and two-dimensional linear dispersions, disorderinduced linear dispersions, asymmetrical linear dispersions, and linear dispersions in topological insulators. The zero-gap state with quadratic dispersions can be obtained in any narrow-band III-V or II-VI semiconductor by either chemical doping or application of external pressure. Oxide-based zero-gap materials are particularly desirable. It is the two-dimensional zero-gap band structure with linear energy dispersion that gives graphene so many fascinating physical properties and its many potential applications at room temperature, and the search for other materials having similar band structures remains a challenge. The two-dimensional organic conductor $\alpha$-(BEDT-TTF $)_{2} \mathrm{I}_{3}$ is one example of such a material. Of particular interest, $\boldsymbol{\alpha}$-(BEDT-TTF) $)_{2} \mathrm{I}_{3}$ exhibits an asymmetrical linear energy dispersion, unique for a twodimensional organic compound. A zero-gap structure with linear energy dispersion can be realized in highly disordered three-dimensional samples displaying quantum linear magnetoresistance. The search for other candidates with three-dimensional band structures having a zero gap and linear energy dispersion will be exciting, and a range of rich physics can be anticipated. In particular, the spin-gapless semiconductors, a new class of zero-gap materials, are expected to show fascinating electronic properties: full spin polarization with tunable charge-carrier concentrations and the generation of polarized photons. It is possible to realize the spin-gapless feature in a wide range of zero-gap and narrow-band materials. Topological insulators, a special class of zerogap materials, have unique spin-related quantum effects and the search for more topological insulators and more quantum effects has become a main focus of research in both materials and condensed physics. The zero-gap materials and their unique band structures are therefore expected to form the basis for future spintronics, electronics and optics.

\section{Acknowledgments}

This work is supported by the Australian Research Council through a Queen Elizabeth II Fellowship (X. L. Wang) and a Discovery project (DP0558753) (X. L. Wang) entitled "The exploration for new materials for spintronics". The authors thank Junfeng Liu and Lei Chen for technical assistance, and Tania Silver for careful editing of the manuscript. C. Zhang acknowledges the support of the ARC Discovery project (DP0879151) entitled "Novel graphene nanostructures: modelling, synthesis, fabrication and characterisation".

\section{References}

[1] I. Žutić, J. Fabian, S. Das Sarma, Rev. Mod. Phys. 76, 323 (2004).

[2] C. Felser, G. H. Fecher, B. Balke, Angew. Chem. Int. Ed. 46, 668 (2007).

[3] T. Dietl, D. D. Awschalom, M. Kaminska, H. Ohno, Spintronics, Elsevier Science (2008).

[4] W. Eerenstein, N. D. Mathur, J. F. Scott, Nature 442, 759 (2006).
[5] R. Ramesh, N. A. Spaldin, Nature Mater. 6, 21 (2007).

[6] R. A. de Groot, F. M. Mueller, P. G. van Engen, K. H. J. Buschow, Phys. Rev. Lett. 50, 2024 (1983).

[7] X. L. Wang, E. Takayama-Muromachi, Phys. Rev. B 72, 064401 (2005).

[8] H. van Leuken, R. A. de Groot, Phys. Rev. Lett. 74, 1171 (1995).

[9] W. E. Pickett, Phys. Rev. B 57, 10613 (1998).

[10] H. Akai, M. Ogura, Phys. Rev. Lett. 97, 026401 (2006).

[11] Y.-M. Nie, X. Hu, Phys. Rev. Lett. 100, 117203 (2008).

[12] I. M. Tsidilkovski, in Electron Spectrum of Gapless Semiconductors, K. von Klitzing ed., Springer Series in Solid-State Sciences Vol. 116, Springer, New York (1996).

[13] A. H. Castro Neto, F. Guinea, N. M. R. Peres, K. S. Novoselov. A. K. Geim, Rev. Mod. Phys. 81, 109 (2009).

[14] W. D. Lawson, S. Nielson, E. H. Putley, A. S. Young, J. Phys. Chem. Sol. 9, 325 (1959).

[15] A. Rogalski, Rep. Prog. Phys. 68, 2267 (2005).

[16] G. Bush, R. Kern, Solid State Physics: 11, 1, Academic, New York (1960).

[17] F. H. Pollak, M. Cardona, C. W. Higginbotham, F. Herman, J. P. Van Dyke, Phys. Rev. B 2, 352 (1970).

[18] S. H. Groves, W. Paul, Phys. Rev. Lett. 11, 194 (1963).

[19] F. Pool, J. Kossut, U. Debska, R. Reifenberger, Phys. Rev. B 35, 3900 (1987).

[20] K. S. Novoselov et al., Science 306, 666 (2004).

[21] K. S. Novoselov et al., Nature 438, 197 (2005).

[22] Y. Zhang, Y.-W. Tan, H. L. Stormer, P. Kim, Nature 438, 201 (2005).

[23] C. Berger et al., Science 312, 1191 (2006).

[24] H. Suzuura, T. Ando, Phys. Rev. Lett. 89, 266603 (2002).

[25] S. V. Morozov et al., Phys. Rev. Lett. 97, 016801 (2006).

[26] D. V. Khveshchenko, Phys. Rev. Lett. 97, 036802 (2006).

[27] E. McCann et al., Phys. Rev. Lett. 97, 146805 (2006).

[28] Q. Yan et al., Nano Lett. 7, 1469 (2007).

[29] Y. S. Zheng, T. Ando, Phys. Rev. B 65, 245420 (2002).

[30] N. M. R. Peres, F. Guinea, A. H. Castro Neto, Phys. Rev. B 73, 125411 (2006).

[31] K. Ziegler, Phys. Rev. Lett. 97, 266802 (2006).

[32] I. L. Aleiner, K. B. Efetov, Phys. Rev. Lett. 97, 236801 (2006).

[33] Y.-W. Son, M. L. Cohen, S. G. Louie, Nature 444, 347 (2006).

[34] L. Pisani, J. A. Chan, B. Montanari, N. M. Harrison, Phys. Rev. B 75, 064418 (2007).

[35] O. V. Yazyev, L. Helm, Phys. Rev. B 75, 125408 (2007).

[36] E. H. Hwang, S. Adam, S. Das Sarma, Phys. Rev. Lett. 98, 186806 (2007).

[37] M. Ezawa, Phys. Rev. B 73, 045432 (2006).

[38] J. Liu, A. R. Wright, C. Zhang, Z. Ma, Appl. Phys. Lett. 93, 041106 (2008).

[39] A. R. Wright, J. C. Cao, C. Zhang, Phys. Rev. Lett. 103, 207401 (2009).

[40] A. B. Kuzmenko, E. van Heumen, F. Carbone, D. van der Marel, Phys. Rev. Lett. 100, 117401 (2008).

[41] C. Zhang, L. Chen, Z.S. Ma, Phys. Rev. B 77, 241402 (2008).

[42] R. R. Nair et al., Science 320, 1308 (2008).

[43] D. Yu, E. M. Lupton, H. J. Gao, C. Zhang, F. Liu, Nano Res. 1, 497 (2008).

[44] E. H. Lieb, Phys. Rev. Lett. 62, 1201 (1989).

[45] J. Liu, Z. Ma, A. R. Wright, C. Zhang, J. Appl. Phys. 103, 103711 (2008).

[46] R. Xu et al. Nature 390, 57 (1997).

[47] A. A. Abrikosov, Phys. Rev. B 58, 2788 (1998).

[48] M. Lee, T. F. Rosenbaum, M.-L. Saboungi, H. S. Schnyders, Phys. Rev. Lett. 88, 066602 (2002).

[49] M. M. Parish, P. B. Littlewood, Nature 426, 162 (2003).

[50] J. S. Hu, T. F. Rosenbaum, Nature Mater. 71, 697 (2008).

[51] H. Kino, T. Miyazaki, J. Phys. Soc. Jpn 75, 034704 (2006).

[52] K. Bender et al., Mol. Cryst. Liq. Cryst. 108, 359 (1984).

[53] N. Tajima, S. Sugawara, M. Tamura, Y. Nishio, K. Kajita, J. Phys. Soc. Jpn 75, 051010 (2006).

[54] N. Tajima, A. Ebina-Tajima, M. Tamura, Y. Nishio, K. Kajita, J. Phys. Soc. Jpn 71, 1832 (2002).

[55] X. L. Wang, Phys. Rev. Lett. 100, 156404 (2008).

[56] X. L. Wang, G. Peleckis, S. X. Dou, C. Zhang, H. Kimura, Adv. Mater. 21, 2196 (2009).

[57] T. C. Ozawa et al., J. Alloys Compd. 388, 1 (2005).

[58] A. Asamitsu, Y. Tomioka, H. Kuwahara, Y. Tokura, Nature 388, 50 (1997).

[59] F. X. Hu, J. Gao, S. X. Wu, Phys. Rev. B 72, 064428 (2005).

[60] D. Yu, E. M. Lupton, M. Liu, W. Liu, F Liu, Nano Res. 1, 56 (2008).

[61] A. Ohtomo, H. Y. Hwang, Nature 427, 423 (2004).

[62] S. Okamoto, A. J. Millis, Nature 428, 630 (2004).

[63] H. Y. Hwang, Mater. Res. Soc. Bull. 31, 28 (2006).

[64] E. Bousquet et al., Nature 452, 732 (2008).

[65] A. Brinkman et al., Nat. Mater. 6, 493 (2007).

[66] N. Reyren et al., Science 317, 1196 (2007).

[67] A. D. Caviglia et al., Nature 456, 624 (2008).

[68] B. A. Bernevig, T. L. Hughes, S.-C. Zhang, Science 314, 1757 (2006).

[69] M. König et al., Science 318, 766 (2007). 
[70] B.A. Bernevig, S.-C. Zhang, Phys. Rev. Lett. 96, 106802 (2006)

[71] C. L. Kane, E. J. Mele, Phys. Rev. Lett. 95, 146802 (2005).

[72] L. Fu, C. L. Kane, E. J. Mele, Phys. Rev. Lett. 98, 106803 (2007).

[73] J. E. Moore, L. Balents, Phys. Rev. B 75, 121306 (2007).

[74] R. Roy, Phys. Rev. B 79, 195321 (2009).

[75] X.-L. Qi, T. L. Hughes, S.-C. Zhang, Phys. Rev. B 78, 195424 (2008).

[76] X. Dai et al., Phys. Rev. B 77125319 (2008).
[77] L. Fu, C. L. Kane, Phys. Rev. B 76, 045302 (2007)

[78] J. C. Y. Teo, L. Fu, C. L. Kane, Phys. Rev. B 78, 045426 (2008).

[79] H. J. Zhang et al., preprint available at http://arxiv.org/abs/0812.1622

[80] Y. Xia et al., preprint available at http://arxiv.org/abs/0812.2078v1

[81] Y. L. Chen et al., preprint available at http://arxiv.org/abs/0904.1829v1

[82] S. C. Zhang, Physics 1, 6 (2008).

[83] A. Shitade et al., Phys. Rev. Lett. 102, 256403 (2009).

\section{Author profiles}

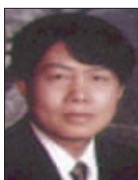

\section{Xiao-Lin Wang}

Xiao-Lin Wang received his BSc and MSc degrees in physics from Shandong University in China and his PhD in materials engineering in 2000 from the University of Wollongong, Australia. He is professor in the Faculty of Engineering, University of Wollongong and group leader of the spintronic and electronic group at the Institute for Superconducting and Electronic Materials at the same institution. His research interests cover the materials and solid-state physics of design for new classes of materials, spintronics, mutiferroics and superconductors.

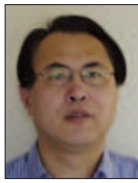

\section{Chao Zhang}

Chao Zhang received his graduate diploma in physics from East China Normal University in 1981, and earned his MA degree in physics at City College of New York, USA in 1984 and his PhD degree in physics at the City University of New York, USA in 1987. He was a postdoctoral fellow at Max-Planck-Institute for Solid State Research in Stuttgart, Germany from 1987 to 1989, and a research associate at TRIUMF in Vancouver, Canada from 1989 to 1992. He is currently a professor in the School of Engineering Physics, University of Wollongong, Australia where he has been since 1993. He holds guest professorships at the Shanghai Institute of Microsystem and Information Technology, Institute of Solid State Physics and Xian Institute of Optics and Precision Mechanics, Chinese Academy of Sciences, and at Tianjin University. His research mainly focuses on the quantum transport and optical properties of nanosystems.

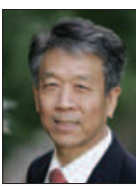

\section{Shi Xue Dou}

Shi Xue Dou joined the University of Wollongong, Australia in 1994. He is an Australian Professorial Fellow at the University of Wollongong and director of the Institute for Superconducting and Electronic Materials. He earned his PhD degree in solid-state electrolytes for fuel cells and oxygen sensors at Dalhousie University in Canada in 1984, and was awarded a DSc degree for his work on high-temperature superconductivity at the University of New South Wales, Australia in 1998. His research interests include materials processing, characterization and applications, superconductors, energy materials and magnetic materials. 